\title{
Computational confirmation of an abnormal Mach reflection wave configuration
}

\author{
Z. M. Hu, ${ }^{1, a)}$ C. Wang, ${ }^{2}$ Y. Zhang, ${ }^{3}$ and R. S. Myong ${ }^{1,3, b)}$ \\ ${ }^{1}$ Research Center for Aircraft Parts Technology (ReCAPT), Gyeongsang National University, \\ Jinju 660-701, South Korea \\ ${ }^{2}$ Key Laboratory of High Temperature Gas Dynamics (LHD), Chinese Academy of Sciences, \\ Beijing 100080, China \\ ${ }^{3}$ School of Mechanical and Aerospace Engineering, Gyeongsang National University, \\ Jinju 660-701, South Korea
}

(Received 14 October 2008; accepted 30 December 2008; published online 29 January 2009)

\begin{abstract}
For the Mach reflection (MR) of symmetric shock waves of opposite families, only the wave configuration of an overall Mach reflection (oMR) consisting of two direct Mach reflections (DiMR + DiMR) is theoretically admissible. For asymmetric shock waves, an oMR composed of a DiMR and an inverse Mach reflection (InMR) is possible if the two slip layers assemble a converging-diverging stream tube, while an oMR including two inverse Mach reflections (InMR + InMR) is absolutely impossible. In this paper, an overall Mach reflection configuration with double inverse MR patterns is computationally confirmed using the computational fluid dynamics technique. The aerodynamic mechanism behind such an abnormal wave pattern is illustrated. Classical two- and three-shock theories are also applied for the theoretical analysis. (C) 2009 American Institute of Physics. [DOI: 10.1063/1.3073006]
\end{abstract}

The shock wave interactions have significant impacts on the performance and reliability of a supersonic/hypersonic aircraft or a winged space shuttle during its re-entry flight. The study on shock interactions can be traced back to 1878 when Ernst Mach found two well known shock wave reflection configurations: a regular reflection (RR) and a Mach reflection (MR), which consists of a Mach stem, an incident shock, a reflected shock, and a slip layer. von Neumann ${ }^{1}$ introduced the criteria for the $\mathrm{RR} \leftrightarrow \mathrm{MR}$ transition of symmetric shock waves: the von Neumann and detachment criteria. Theoretically, MR is impossible below the former, while RR is inadmissible beyond the latter. Both RR and MR are admissible between the criteria. Related research can still be found in the recent literature. ${ }^{2-6}$

In the present work, an abnormal MR configuration of asymmetric shock waves is computationally confirmed over a double-wedge geometry (Fig. 1) posed in a hypersonic flow. Such an overall Mach reflection (oMR) configuration, which is theoretically impossible, ${ }^{7,8}$ consists of two inverse Mach reflections (InMRs) and is denoted as oMR(InMR + InMR). In an InMR the slip layer and the reflection plane form a diverging stream tube. On the contrary, in a direct Mach reflection (DiMR) they form a converging stream tube. The physical mechanism behind such an oMR(InMR $+\mathrm{InMR})$ is found to be the diverging-converging-diverging stream tube generated due to shock wave-slip layer interactions following the oMR. Numerical studies on the similar problem were conducted elsewhere. ${ }^{2,3,6,9,10}$ However, the existence of an abnormal oMR(InMR + InMR) wave configura-

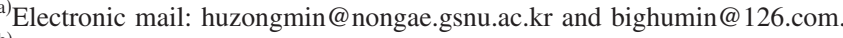
${ }^{b)}$ Author to whom correspondence should be addressed. Electronic mail: myong@gnu.ac.kr.
}

tion, to the best of our knowledge, has never been reported until the present work.

Figure 2 schematically shows the MR configuration for two shock waves of opposite families. The previous studies reached a conclusion that an overall MR configuration, which is composed of two DiMRs, i.e., oMR(DiMR + DiMR), is the only theoretically admissible solution for a symmetric MR when $\theta_{1}=\theta_{2}$. An oMR $(\operatorname{InMR}+\mathrm{DiMR})$ will be possible only if the two slip layers (marked by $s 1$ and $s 2$ ) assemble a converging-diverging stream tube for an asymmetric reflection $\left(\theta_{1} \neq \theta_{2}\right){ }^{7,8}$ Here, the converging-diverging stream tube can bridge the locally subsonic flow downstream of the Mach stem (denoted by $m$ ) with the overall supersonic flow. The wave pattern in Fig. 2 presents an oMR(DiMR + DiMR), while in Fig. 3 an oMR(InMR+DiMR) is confirmed by an inviscid computational fluid dynamics (CFD) computation.

The two slip layers of an oMR(InMR + InMR) wave pattern form a diverging stream tube that cannot provide that "bridge." However, as stated by Henderson and Menikoff, ${ }^{11}$ the local downstream boundary conditions can affect the solution due to the fact that the wave pattern, in steady state, must be compatible with the global flow. The flow domain of the present interest is shown in Fig. 1 for the inviscid interaction of hypersonic flows and double-wedge geometries. The shock wave interaction phenomena depend on the relevant parameters, which are, under the inviscid flow hypothesis, the freestream Mach number $M_{\infty}$, the ratio of the specific heats $\gamma$, the wedge length, and the wedge angles $\theta_{1}$ and $\theta_{2}$. The geometric dimensions are normalized by the first wedge length, $L_{1}$. The computational domain surrounded by the dashed-dotted rectangle as shown in Fig. 1 is used for computational cheapness. The distance of the first 


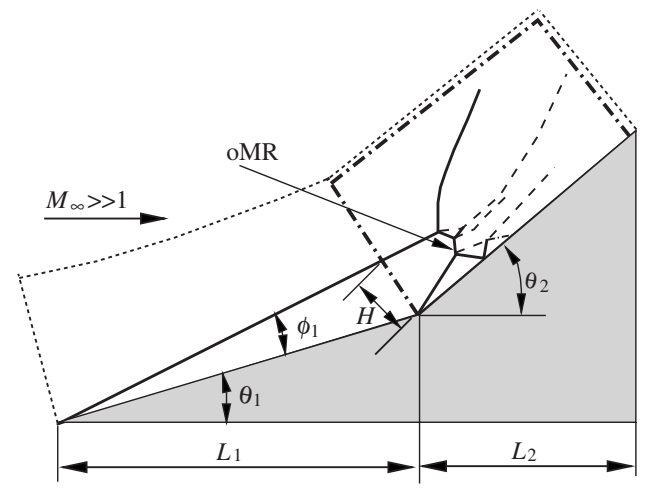

FIG. 1. Flow geometry and the simplified computational domain.

leading shock wave to the wedge corner can be analytically defined as

$$
\frac{H}{L_{1}}=\frac{\sin \left(\phi_{1}-\theta_{1}\right) \cos \left(\phi_{1}-\theta_{1}\right)}{\cos \left(\theta_{2}-\phi_{1}\right)},
$$

where $\phi_{1}$ is the shock angle over the first wedge.

For numerical algorithms in the present study, Euler equations for a perfect gas with $\gamma=1.4$ are spatially discretized using the second-order dispersion controlled dissipative (DCD) scheme. ${ }^{12,13}$ The principle of DCD is to suppress nonphysical oscillation across strong discontinuities by making use of the intrinsic dispersion characteristics of the modified equations instead of adding artificial viscosity. A thirdorder Runge-Kutta scheme is used for temporal integration. Pressure-deflection polar diagrams for shock interaction are also applied for the theoretical analysis. Briefly, the shock polar represents the locus of all flow states that can be obtained by passing through a shock wave of a given flow Mach number. The entire region behind a planar shock wave

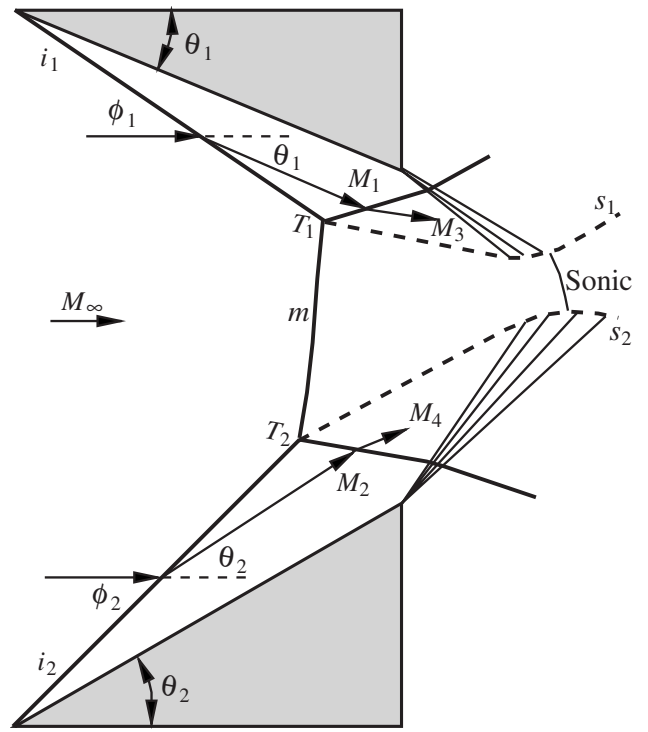

FIG. 2. Sketch of a MR configuration.

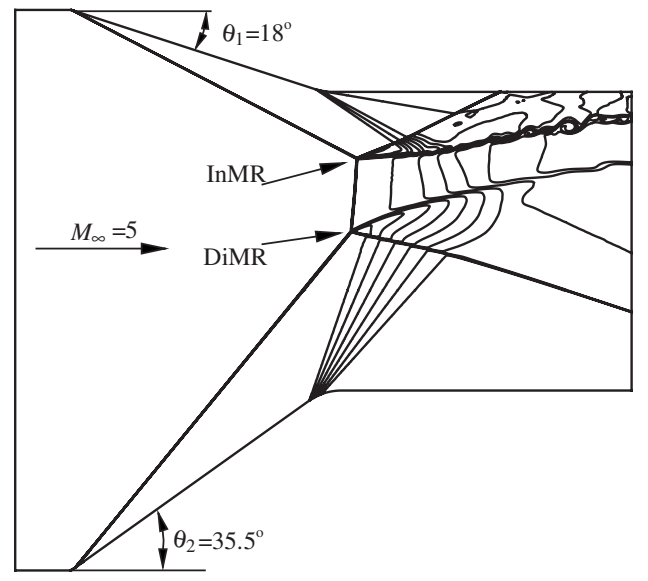

FIG. 3. CFD confirmation of an oMR(InMR+DiMR) reflection configuration (grid: $1601 \times 1601)$.

is then represented by a single point on a $p-\theta$ diagram. The flow deflection angle $\theta$ and the pressure ratio $\xi$ across an oblique shock wave can be respectively related to the Mach number $M$ ahead of the shock wave and the shock angle $\phi$ as follows:

$$
\begin{aligned}
& \theta=\theta(\gamma, M, \phi)=\arctan \left\{\frac{2 \cot \phi\left(M^{2} \sin ^{2} \phi-1\right)}{M^{2}(\cos 2 \phi+\gamma)+2}\right\}, \\
& \xi=\xi(\gamma, M, \phi)=1+\frac{2 \gamma}{\gamma+1}\left(M^{2} \sin ^{2} \phi-1\right) .
\end{aligned}
$$

Here, $\phi$ denotes the shock angle, and $\arcsin (1 / M) \leq \phi$ $\leq \pi / 2$. With the above equations, the pressure jump across a shock wave can be plotted against the flow deflection angle.
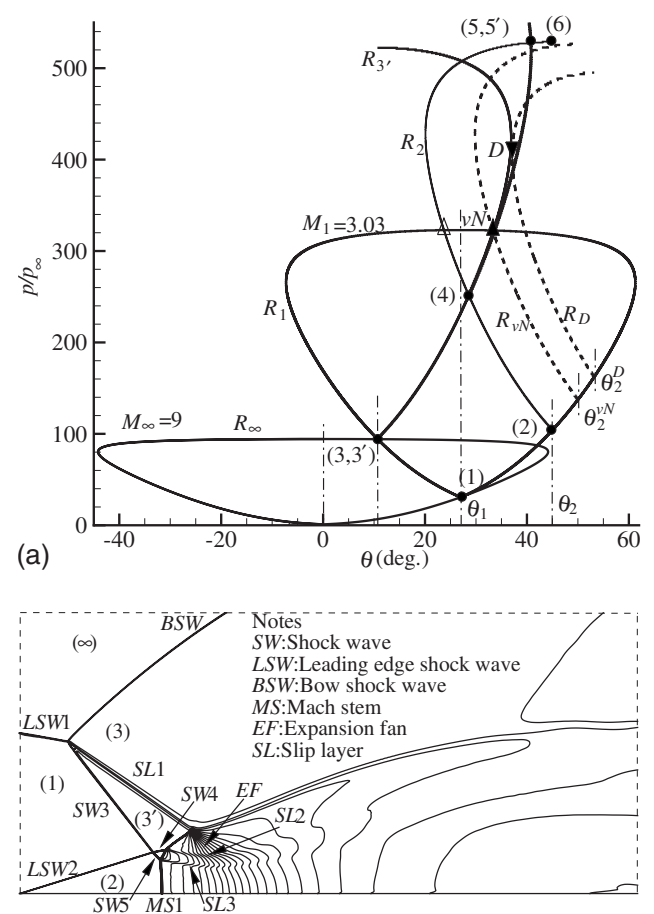

(b)

FIG. 4. (a) Shock polar combination. (b) The computed wave configuration $\left(M_{\infty}=9, \theta_{1}=27^{\circ}, \theta_{2}=45^{\circ}\right.$, and grid: $\left.651 \times 551\right)$. 
The first case is for the interaction of a $M_{\infty}=9$ hypersonic flow with a double-wedge-like geometry, where $\theta_{1}$ $=27^{\circ}$ and $\theta_{2}=45^{\circ}$. The shock polar combination, along with the computational wave configuration, is shown in Fig. 4. The von Neumann and detachment criteria ${ }^{7}$ corresponding to fixed flow conditions of $(1)$ and $\left(3,3^{\prime}\right)$ are additionally plotted as the dashed lines in Fig. 4(a). Here, LSW1 and LSW2 denote the leading shock waves emanating from both edges, respectively. SL denotes the slip layer initiates from a triple point, while MS and BSW denote a Mach stem and a bow shock wave. The detailed explanation about the wave structure can be found in a former article. ${ }^{9}$ It is not surprising that the shock waves of opposite families, LSW2 and SW3, go on a regulation interaction at point (4) because $\theta_{2}$ is smaller than the corresponding von Neumann criterion $\theta_{2}^{\mathrm{vN}}$. This is proven by both the shock polar analysis and the computation. It should be noted that the computational pressure field in region $\left(3^{\prime}\right)$, as shown in Fig. 4(b), is slightly nonuniform due to the complex interaction of shock waves in the presence of subsonic zone, i.e., region (3). However, the nonuniformity is insignificant (2\%-3\% in pressure) and does not essentially influence the results.

After slightly increasing the wedge angle $\theta_{2}$ to $45.5^{\circ}$, the shock polar combination does not change much. The shock loci $R_{2}$ and $R_{3}$, still intersect inside the $R_{1}$ polar. Therefore, the solution of the shock interaction between LSW2 and SW3 should theoretically be a regular reflection as denoted by points $[4]$ and $\left[5,5^{\prime}\right]$ on the polar combination. However, the computation, as plotted in Fig. 5(b), finally comes around to a MR as given by the solution series $\left(4,4^{\prime}\right),\left(5,5^{\prime}\right),\left(6,6^{\prime}\right)$ on the shock polar combination in Fig. 5(a). It is the triple point of the local MR wave pattern connecting to the wall in Fig. 4(b) that collides with the regular interaction point [4] and changes it into a MR. The mechanism of the advanced $\mathrm{RR} \rightarrow \mathrm{MR}$ transition can be found in an early study. ${ }^{10}$ Most surprisingly, the solutions $\left(4,4^{\prime}\right)$ and $\left(5,5^{\prime}\right)$ correspond to an oMR(InMR + InMR) wave configuration which should be theoretically impossible. ${ }^{7}$ The further computation on a grid of higher density reaches the same wave pattern, as shown in Fig. 5(c), which indicates grid independence. KelvinHelmholtz instability ${ }^{14}$ of slip layer is captured in the far field and has an insignificant influence on the shock wave interaction pattern. Here, the iterative convergence is considered to be achieved if the maximum pressure along the wedge surface and its location remain stationary within a certain iterations.

The pair of slip layers, i.e., SL2 and SL3 as shown in Fig. 5(b) or Fig. 5(c), assembles a diverging stream tube slightly downstream of the Mach stem MS2 of the oMR(InMR + InMR) wave configuration. Therefore, additional boundary conditions should be imposed downstream of the interaction to stabilize such a theoretically unstable wave pattern. In Fig. 5(b) or Fig. 5(c), the reflected shock wave SW6 impinges on the slip layer SL3 and makes the latter turn upward. At the same time, shock wave SW4 is reflected from slip layer SL1 and sequentially turns SL2 downward. In consequence, SL2 and SL3 move close to each other and assemble a converging stream tube. Further, the expansion fan (EF) turns SL2 upward and let the stream tube
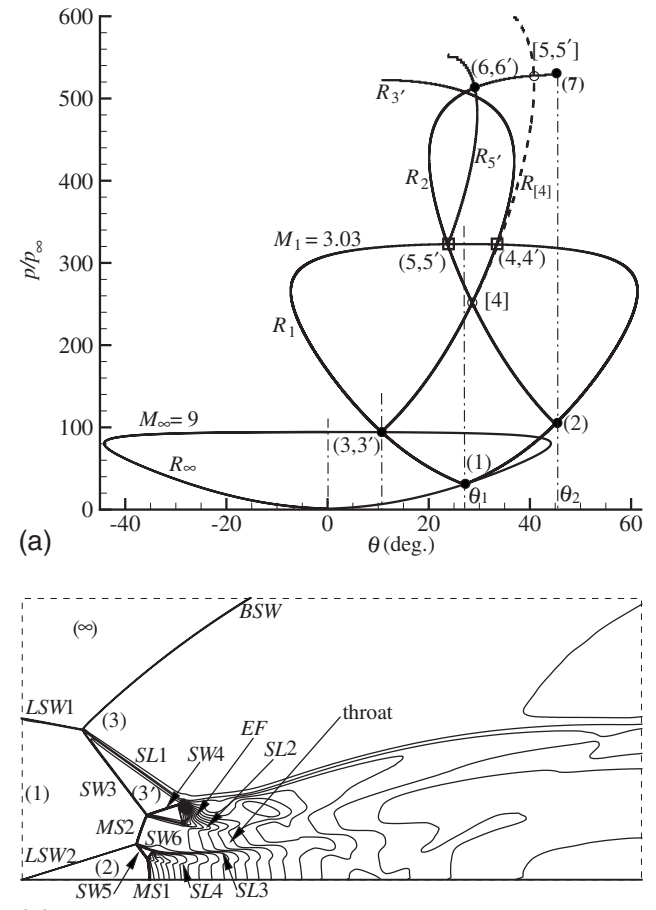

(b)

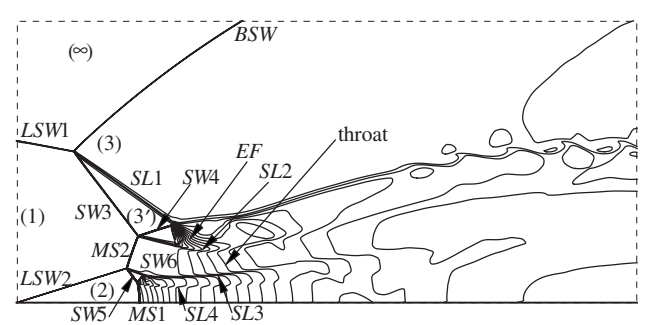

(c)

FIG. 5. (a) Shock polar combination and the computed wave configurations: (b) grid $651 \times 551$ and $(\mathrm{c})$ grid $1001 \times 901\left(M_{\infty}=9, \theta_{1}=27^{\circ}\right.$, and $\left.\theta_{2}=45.5^{\circ}\right)$.

diverge again. Finally, the resulted diverging-convergingdiverging steam tube between SL2 and SL3 serves for the physical mechanism for a steady abnormal wave pattern of oMR(InMR + InMR).

In conclusion, an abnormal wave configuration with a pair of inverse MRs which is theoretically inadmissible has been confirmed by CFD computations. The physical mechanism behind it includes two steps of wave dynamics. First, the $\mathrm{RR} \rightarrow \mathrm{MR}$ transition is advanced by a transverse wave emanating from a triple point impinging off the wedge surface. Consequently, the resulted MR wave configuration is stabilized by the converging stream tube formed in virtue of the shock wave-slip layer interaction from the downstream field. Computations indicate that this exceptional wave pattern can only occur inside a very small scope of wedge angle for a given freestream flow Mach number. A validation experimental work may be needed to confirm the new finding in the future.

This work was supported by Korea Research Foundation under Grant No. KRF-2005-005-J09901. One of the authors (C.W.) acknowledges support provided by the National Natural Science Foundation of China (Grant No. 10602059). 
${ }^{1}$ J. von Neumann, "Refraction, interaction and reflection of shock waves," NAVORD Report No. 203-45, 1943.

${ }^{2}$ J. Olejniczak, W. J. Wright, and G. V. Candler, "Numerical study of inviscid shock interactions on double-wedge geometries," J. Fluid Mech. 352, 1 (1997).

${ }^{3}$ G. Ben-Dor, E. I. Vasilev, T. Elperin, and A. V. Zenovich, "Self-induced oscillations in the shock wave flow pattern formed in a stationary supersonic flow over a double wedge," Phys. Fluids 15, L85 (2003).

${ }^{4}$ C. A. Mouton and H. G. Hornung, "Mach stem height and growth rate predictions," AIAA J. 45, 1977 (2007).

${ }^{5}$ C. A. Mouton, "Transition between regular reflection and Mach reflection in the dual-solution domain," Ph.D. thesis, California Institute of Technology, Pasadena, California, 2007.

${ }^{6}$ G. Ben-Dor, T. Elperin, and E. I. Vasilev, "Shock wave-induced extremely high temporal and spatial oscillating pressure peaks," J. Aerosp. Eng. 222, 653 (2008)

${ }^{7} \mathrm{H}$. Li, A. Chpoun, and G. Ben-Dor, "Analytical and experimental investigations of the reflection of asymmetric shock waves in steady flows," J. Fluid Mech. 390, 25 (1999).

${ }^{8}$ M. S. Ivanov, G. Ben-Dor, A. N. Kudryavtsev, and D. V. Khotyyanovsky,
"The reflection of asymmetric shock waves in steady flows: A numerical investigation," J. Fluid Mech. 469, 71 (2002).

${ }^{9}$ Z. M. Hu, R. S. Myong, C. Wang, T. H. Cho, and Z. L. Jiang, "Numerical study of the oscillations induced by shock/shock interaction in hypersonic double-wedge flows," Shock Waves 18, 41 (2008).

${ }^{10}$ Z. M. Hu, R. S. Myong, M. S. Kim, and T. H. Cho, "Downstream flow conditions effects on the RR MR transition of asymmetric shock waves in steady flows," J. Fluid Mech. 620, 43 (2009).

${ }^{11}$ L. F. Henderson and R. Menikoff, "Triple shock entropy theorem and its consequences," J. Fluid Mech. 366, 179 (1998).

${ }^{12}$ Z. L. Jiang, K. Takayama, and Y. S. Chen, "Dispersion conditions for non-oscillatory shock-capturing schemes and its applications," Comput. Fluid Dyn. J. 2, 137 (1995).

${ }^{13} \mathrm{Z}$. L. Jiang, "On the dispersion-controlled principles for non-oscillatory shock-capturing schemes," Acta Mech. Sin. 20, 1 (2004).

${ }^{14}$ A. Rikanati, O. Sadot, G. Ben-Dor, D. Shvarts, T. Kuribayashi, and K. Takayama, "Shock-wave Mach-reflection slip-stream instability: A secondary small-scale turbulent mixing phenomenon," Phys. Rev. Lett. 96, 174503 (2006) 